\title{
ON THE CARTESIAN PRODUCTS OF LINDELÖF SPACES WITH ONE FACTOR HEREDITARILY LINDELÖF
}

\author{
K. ALSTER
}

(Communicated by Dennis R. Burke)

\begin{abstract}
E. Michael asked the following question: Is there a space $X$ such that $Y \times X$ is Lindelöf for every hereditarily Lindelöf space $Y$ but $X^{2}$ is not. The aim of this paper is to present a construction that provides such an example.
\end{abstract}

In this paper we construct for every $n \in N$ a space $X_{n}$ such that for every hereditarily Lindelöf space $Y, Y \times X_{n}^{n}$ is Lindelöf and $X_{n}^{n+1}$ is not. Moreover, we also obtain, using the same technique, a space $X_{\omega}$ such that for every $n \in N$ and every hereditarily Lindelöf space $Y, Y \times X_{\omega}^{n}$ is Lindelöf but $X_{\omega}^{\omega}$ is not.

Let us recall that another example of $X_{\omega}$ was presented in [A2, A3]. The present construction is much simpler than the previous one.

For related results and constructions see [A5, T, A6, A4].

Our topological terminology follows [E]. In particular, if $M$ is a subspace of a topological space $X$ then the symbol $X_{M}$ stands for the set $X$ with a new topology generated by $\mathscr{T}=\{U \subset X: U$ is open in $X\} \cup\{\{x\}: x \in X \backslash M\}$. In the sequel $Q$ and $N$ stand for the set of all rational and natural numbers respectively. Let us denote by $\mathscr{B}$ a countable base for $Q$ consisting of open intervals. If $x \in Q$ then put $\mathscr{B}_{x}=\{B \in \mathscr{B}: x \in B\}$. The symbols $\omega$ and $\omega_{1}$ denote the first infinite ordinal number and the first uncountable ordinal number, respectively.

The projection of $Q^{\omega_{1}}$ onto $Q^{T}$ where $T \subset \omega_{1}$ will be denoted by $P_{T}$. If $\alpha$ is an ordinal number then we shall identify $\alpha$ with the set of its predecessors. If $\alpha<\beta$, and $x \in Q^{\beta}$ then let us denote $P_{\alpha}(x)$ by $x \mid \alpha$.

Example. For all $n \in N$ there is $X_{n}$ such that for every hereditarily Lindelöf space $Y, Y \times\left(X_{n}\right)^{n}$ is Lindelöf but $\left(X_{n}\right)^{n+1}$ is not. Moreover, there is also $X_{\omega}$ such that for all $n \in N, Y \times\left(X_{\omega}\right)^{n}$ is Lindelöf but $\left(X_{\omega}\right)^{\omega}$ is not.

We now consider the proof of the example. There exists a family $\left\{A_{\alpha}: 1 \leq\right.$ $\left.\alpha<\omega_{1}\right\}$ such that

(1) $A_{\alpha}$ is a countable set consisting of strictly increasing sequences of rational numbers of length $\alpha$ for $1 \leq \alpha<\omega_{1}$,

Received by the editors April 24, 1990.

1991 Mathematics Subject Classification. Primary 54B10.

Key words and phrases. Lindelöf space, hereditarily Lindelöf, Cartesian product.

Research supported by Polish scientific grant R.P.I. 10. 
(2) if $\alpha<\beta<\omega_{1}$ then $P_{\alpha}\left(A_{\beta}\right)=A_{\alpha}$,

(3) if $a=(a(\lambda))_{\lambda<\alpha} \in A_{\alpha}$ then $a$ is a continuous function from $\alpha$ into $Q$ and $\sup \{a(\lambda): \lambda<\alpha\}$ is a rational number (see [J, p. 91], the construction of the Aronszajn tree).

Let us attach to $a \in A_{\alpha}$, for $1 \leq \alpha<\omega_{1}, x_{a} \in Q^{\omega_{1}}$ such that

$$
x_{a}(\beta)= \begin{cases}a(\beta) & \text { if } \beta<\alpha, \\ \sup \{a(\lambda): \lambda \leq \alpha\} & \text { if } \beta \geq \alpha .\end{cases}
$$

Put $Z_{\alpha}=\left\{x \in Q^{\omega_{1}}\right.$ : there is a $a \in A_{\alpha}$ such that $\left.x=x_{a}\right\}$. If $x \in \bigcup\left\{Z_{\alpha}: 1 \leq\right.$ $\left.\alpha<\omega_{1}\right\}$ then $\alpha(x)$ is such an ordinal number that $x \in Z_{\alpha(x)}$.

Let $\left\{S_{i}: i \leq n+1\right\}$ be a cover of $\omega_{1}$ consisting of pairwise disjoint stationary sets and put $K_{i}=\bigcup\left\{S_{j}: j \neq i\right\}$ for all $i \leq n+1$.

If $S$ is a subset of $\omega_{1}$ then put $X(S)=\bigcup\left\{Z_{\alpha}: \alpha \in S\right\}$.

The desired space $X_{n}$ is defined by $X_{n}=\bigoplus_{i \leq n+1}\left(X\left(\omega_{1}\right)_{X\left(K_{i}\right)}\right)$ where $X\left(\omega_{1}\right)$ and $X\left(K_{i}\right)$ are subspaces of $Q^{\omega_{1}}$.

In case of $X_{\omega}$ we have to consider infinite partition $\left\{S_{n}: n \in N\right\}$ of $\omega_{1}$ consisting of stationary sets and put

$$
X_{\omega}=\bigoplus_{i=1}^{\infty}\left(X\left(\omega_{1}\right)\right)_{X\left(K_{i}\right)}, \quad \text { where } K_{i}=\bigcup\left\{S_{j}: j \neq i\right\} .
$$

Since $D=\left\{x=\left(x_{i}\right)_{i=1}^{n+1} \in \prod_{i=1}^{n+1}\left(X\left(\omega_{1}\right)_{X\left(K_{i}\right)}\right)\right.$ : the coordinates of $x$ are equal $\}$ is a discrete closed and uncountable subset of $\left(X_{n}\right)^{n+1}$, we infer that $\left(X_{n}\right)^{n+1}$ is not Lindelöf.

In order to prove that $Y \times\left(X_{n}\right)^{n}$ is Lindelöf for every hereditarily Lindelöf space $Y$ we shall need

Lemma 1. If $S$ is a stationary subset of $\omega_{1}$ then $Y \times(X(S))^{n}$ is Lindelöf for every hereditarily Lindelöf space $Y$.

Proof. Let $Y$ be a hereditarily Lindelöf space and $\mathscr{U}$ an open cover of $Y \times$ $(X(S))^{n}$. If $x=x_{a} \in Z_{\alpha}$, where $a \in A_{\alpha}, x_{a}(\alpha) \in B \in \mathscr{B}$, and $\alpha<\lambda$, then put

$$
F(x, B, \lambda)=\left(\prod_{\beta<\omega_{1}} F_{\beta}\right) \cap X\left(\omega_{1}\right),
$$

where

$$
F_{\beta}= \begin{cases}\{x(\beta)\} & \text { if } \beta \leq \alpha, \\ B & \text { if } \beta=\lambda, \\ Q & \text { otherwise. }\end{cases}
$$

For $x=\left(x_{1}, \ldots, x_{n}\right) \in(X(S))^{n}$ and $\prod_{i=1}^{n} B_{i} \in(\mathscr{B})^{n}$, where $x_{i} \in Z_{\alpha\left(x_{i}\right)}$ and $x_{i}\left(\alpha\left(x_{i}\right)\right) \in B_{i}$ for all $i \leq n$ put $A\left(x, \prod_{i=1}^{n} B_{i}\right)=\{y \in Y: \exists$ open $H_{y}, \sup \left\{\alpha\left(x_{i}\right): i \leq n\right\}<\lambda(y)<\omega_{1}$, and $U \in \mathscr{U}$ such that $y \in H_{y}$ and $\left.H_{y} \times \prod_{i=1}^{n}\left(F\left(x_{i}, B_{i}, \lambda(y)\right)\right) \subset U\right\}$.

We can assume, without loss of generality, that $\lambda(y)$ for $y \in A\left(x, \prod_{i=1}^{n} B_{i}\right)$ is as small as possible. Since $\left\{y \in A\left(x, \prod_{i=1}^{n} B_{i}\right): \lambda(y) \leq \beta\right\}$ is open for all $\beta<\omega_{1}$ and $A\left(x, \prod_{i=1}^{n} B_{i}\right)$ is Lindelöf as a subspace of $Y$, we infer that if 
$A\left(x, \prod_{i=1}^{n} B_{i}\right) \neq \varnothing$ then $\sup \left\{\lambda(y): y \in A\left(x, \prod_{i=1}^{n} B_{i}\right)\right\}$ is less than $\omega_{1}$. Put

$$
\lambda\left(x, \prod_{i=1}^{n} B_{i}\right)=\left\{\begin{array}{l}
\sup \left\{\lambda(y): y \in A\left(x, \prod_{i=1}^{n} B_{i}\right)\right\} \quad \text { if } A\left(x, \prod_{i=1}^{n} B_{i}\right) \neq \varnothing, \\
0 \quad \text { otherwise. }
\end{array}\right.
$$

Using again the Lindelöf property of $A\left(x, \prod_{i=1}^{n} B_{i}\right)$ we can find $\left\{y_{j}: j \in\right.$ $N\} \subset A\left(x, \prod_{i=1}^{n} B_{i}\right)$ such that $\bigcup\left\{H_{y_{j}}: j \in N\right\}=A\left(x, \prod_{i=1}^{n} B_{i}\right)$, where $H_{y_{i}}$ was defined in connection with $A\left(x, \prod_{i=1}^{n} B_{i}\right)$.

Let $C$ be a subset of all countable ordinal numbers satisfying the following conditions:

(a) If $\alpha \in C$ then there is a sequence $\left(\alpha_{n}\right)_{n=1}^{\infty}$ in $S$ converging to $\alpha$; we do not require that $\alpha \in S$.

(b) If $\alpha \in C$ then for all $\left(\beta_{1}, \ldots, \beta_{n}\right) \in(S \cap \alpha)^{n}, x=\left(x_{1}, \ldots, x_{n}\right) \in$ $\prod_{i=1}^{n} Z_{\beta_{i}}$ and $\prod_{i=1}^{n} B_{i} \in(\mathscr{B})^{n}$ where $\left(x_{i}\left(\beta_{i}\right)\right)_{i=1}^{n} \in \prod_{i=1}^{n} B_{i}, \lambda\left(x, \prod_{i=1}^{n} B_{i}\right)<\alpha$.

In order to continue the proof of Lemma 1 we need

Claim 1. $C$ is closed and unbounded in $\omega_{1}$.

Proof. Observe that $C$ is closed. Hence the proof will be finished if we show that $C$ is unbounded. Fix $\beta<\omega_{1}$. There is $\beta_{0} \in S \backslash \beta$. Put

$$
\begin{array}{r}
\beta_{1}^{\prime}=\sup \left\{\lambda\left(x, \prod_{i=1}^{n} B_{i}\right): x=\left(x_{i}\right)_{i=1}^{n} \in\left(\bigcup\left\{Z_{\alpha}: \alpha \in S \cap\left(\beta_{0}+1\right)\right\}\right)^{n},\right. \\
\left.\prod_{i=1}^{n} B_{i} \in \prod_{i=1}^{n} \mathscr{B}_{x_{i}\left(\alpha\left(x_{i}\right)\right)}\right\}
\end{array}
$$

and choose $\beta_{1} \in S \backslash \beta_{1}^{\prime}$. If $\beta_{j}$ is defined then put

$$
\begin{array}{r}
\beta_{j+1}^{\prime}=\sup \left\{\lambda\left(x, \prod_{i=1}^{n} B_{i}\right): x=\left(x_{i}\right)_{i=1}^{n} \in\left(\bigcup\left\{Z_{\alpha}: \alpha \in S \cap\left(\beta_{j}+1\right)\right\}\right)^{n},\right. \\
\left.\prod_{i=1}^{n} B_{i} \in \prod_{i=1}^{n} \mathscr{B}_{x_{i}\left(\alpha\left(x_{i}\right)\right)}\right\}
\end{array}
$$

and choose $\beta_{j+1} \in S \backslash \beta_{j+1}^{\prime}$. Then $\gamma=\sup \left\{\beta_{j}: j \in N\right\} \in C$.

Since $S$ is a stationary subset in $\omega_{1}$ and $C$ is closed and unbounded in $\omega_{1}$, there is $\alpha \in S \cap C$. Put

$$
\begin{aligned}
\mathscr{D}= & \left\{H_{y_{i}} \times \prod_{j=1}^{n} F\left(x_{j}, B_{j}, \lambda\left(y_{j}\right)\right): y_{i} \in A\left(x, \prod_{j=1}^{n} B_{j}\right), i \in N,\right. \\
& \left.\prod_{j=1}^{n} B_{j} \in \prod_{j=1}^{n} \mathscr{B}_{x_{j}}\left(\alpha\left(x_{j}\right)\right), \text { and } x=\left(x_{1}, \ldots, x_{n}\right) \in\left(\bigcup\left\{Z_{\gamma}: \gamma \in S \cap \alpha\right\}\right)^{n}\right\} .
\end{aligned}
$$

Observe that $\mathscr{D}$ is a countable family that refines $\mathscr{U}$. In order to finish the proof of Lemma 1 it is enough to show that

Claim 2. $\cup \mathscr{D}=Y \times(X(S))^{n}$.

Proof. Let $(y, x)$ be an arbitrary point of $Y \times(X(S))^{n}$. We shall consider two cases: 
Case 1. $x \in\left(\bigcup\left\{Z_{\gamma}: \gamma \in S \cap(\alpha+1)\right\}\right)^{n}$.

Case 2. Case 1 does not hold.

Proof of Case 1. There are an open subset $y \in H$ of $Y, U \in \mathscr{U}$, finite subsets $T_{i}$ of $\omega_{1}$, and $B_{\gamma} \in \mathscr{B}$ for all $\gamma \in T_{i}$ and $i \leq n$ such that

$$
(y, x) \in H \times \prod_{i=1}^{n}\left(P_{T_{i}}^{-1}\left(\prod_{\gamma \in T_{i}} B_{\gamma}\right)\right) \cap(X(S))^{n} \subset U .
$$

Put $T_{i}=T_{i, 1} \cup T_{i, 2}$, where $T_{i, 1}=T_{i} \cap \alpha\left(x_{i}\right)$ and $T_{i, 2}=T_{i} \backslash T_{i, 1}$ for all $i \leq n$. Since the coordinates of $x_{i}$ for $\gamma \geq \alpha\left(x_{i}\right)$ are the same, we can assume, without loss of generality, that $T_{i, 2}$ is not empty and that there is $B_{i} \in \mathscr{B}$ such that $B_{\gamma}=B_{i}$ for all $\gamma \in T_{i, 2}$ and $i \leq n$.

The sequence $\left(x_{i}(\gamma)\right)_{\gamma<\alpha\left(x_{i}\right)}$ converges to $x_{i}\left(\alpha\left(x_{i}\right)\right)$ so there is $\sup T_{i, 1}<\gamma_{i} \leq$ $\alpha\left(x_{i}\right)$ such that $\gamma_{i} \in S \cap \alpha$ and $x_{i}\left(\gamma_{i}\right) \in B_{i}$ for all $i \leq n$. If $\lambda_{i}=\sup T_{i, 2}, x_{i}=$ $x_{a_{i}}$, and $\lambda=\sup \left\{\lambda_{i}: i=1,2, \ldots, n\right\}$ then $(y, x) \in H \times \prod_{i=1}^{n} F\left(x_{a_{i} \mid y_{i}}, B_{i}, \lambda\right)$. Since elements of $X(S)$ are increasing sequences and $B_{i}$ are intervals for all $i \leq n$, we infer that

$$
\begin{aligned}
(y, x) & \in H \times \prod_{i=1}^{n} F\left(x_{a_{i} \mid \gamma_{i}}, B_{i}, \lambda\right) \\
& \subset H \times \prod_{i=1}^{n}\left(p_{T_{i}}^{-1}\left(\prod_{\gamma \in T_{i}} B_{\gamma}\right)\right) \cap(X(S))^{n} \subset U .
\end{aligned}
$$

From the last fact it follows that

$$
y \in A\left(\left(x_{a_{1} \mid \gamma_{1}}, \ldots, x_{a_{n} \mid \gamma_{n}}\right), \prod_{i=1}^{n} B_{i}\right) .
$$

Observe that since $\gamma_{i}<\alpha$ for all $i \leq n$, we have

$$
\left(x_{a_{1} \mid \gamma_{1}}, \ldots, x_{a_{n} \mid \gamma_{n}}\right) \in\left(\bigcup\left\{Z_{\gamma}: \gamma \in S \cap \alpha\right\}\right)^{n} .
$$

From (5) it follows that there is

$$
y_{k} \in A\left(\left(x_{a_{1} \mid y_{1}}, \ldots, x_{a_{n} \mid \gamma_{n}}\right), \prod_{i=1}^{n} B_{i}\right)
$$

such that

$$
\left(y,\left(x_{a_{1} \mid \gamma_{1}}, \ldots, x_{a_{n} \mid y_{n}}\right)\right) \in H_{y_{k}} \times \prod_{i=1}^{n} F\left(x_{a_{i} \mid y_{i}}, B_{i}, \lambda\left(y_{k}\right)\right) .
$$

Since $x_{i}\left|\gamma_{i}=x_{a_{i} \mid \gamma_{i}}\right| \gamma_{i}$ and for all $\gamma_{i}<\gamma<\omega_{1}, x_{i}(\gamma) \in B_{i}$ and $i \leq n$, we infer by (7) that $(y, x) \in H_{y_{k}} \times \prod_{i=1}^{n} F\left(x_{a_{i} \mid \gamma_{i}}, B_{i}, \lambda\left(y_{k}\right)\right)$. This completes the proof of Case 1.

Proof of Case 2. Let use assume that $x=\left(x_{1}, \ldots, x_{n}\right)$ is an arbitrary point of $(X(S))^{n}$ such that $x_{i}=x_{a_{i}}$. Then let $z=\left(z_{1}, \ldots, z_{n}\right)$ be such that

$$
z_{i}= \begin{cases}x_{i} & \text { if } x_{i} \in U\left\{Z_{\gamma}: \gamma \in S \cap(\alpha+1)\right\}, \\ x_{a_{i} \mid \alpha} & \text { otherwise. }\end{cases}
$$


Then from Case 1 it follows that there is $D=D_{1} \times D_{2} \in \mathscr{D}$ containing $(y, z)$ where $D_{1} \subset Y, D_{2}=\prod_{i=1}^{n} D_{2, i}$, and $D_{2, i} \subset X(S)$ for all $i \leq n$. Since $\alpha \in C$, we infer that

$$
P_{\alpha}^{-1} P_{\alpha}\left(D_{2, i}\right)=D_{2, i} \text { for all } i \leq n .
$$

Since $z_{i} \mid \alpha=x_{i}$ for all $i \leq n$, we conclude, applying (8), that $(y, x) \in D$. This completes the proof of Lemma 1.

Now we are in a position to prove

Lemma 2. If $S$ is a stationary subset of $\omega_{1}$ then for every hereditarily Lindelöf space $Y$ the product $Y \times\left(X\left(\omega_{1}\right)_{X(S)}\right)^{n}$ is Lindelöf.

Proof. Suppose that $Y$ is a hereditarily Lindelöf space. We shall prove Lemma 3 by induction with respect to $n$. In order to simplify the induction we shall assume that $\left(X\left(\omega_{1}\right)_{X(S)}\right)^{0}$ is a one point set. If $n=0$ then there is nothing to prove. Let us suppose that $Y \times\left(X\left(\omega_{1}\right)_{X(S)}\right)^{n}$ is Lindelöf and that $\mathscr{U}$ is an open cover of $Y \times\left(X\left(\omega_{1}\right)_{X(S)}\right)^{n+1}$ consisting of the sets of the form $H \times$ $\prod_{i=1}^{n+1} P_{T_{i}}^{-1}\left(\prod_{\gamma \in T_{i}} B_{\gamma}\right) \cap\left(X\left(\omega_{1}\right)\right)^{n+1}$ where $B_{\gamma} \in \mathscr{B}$ for all $\gamma \in T_{i}$ and $T_{i}$ is a finite subset of $\omega_{1}$ for $i \leq n+1$. Then by Lemma 1 there is a countable subfamily $\mathscr{U}^{\prime}$ of $\mathscr{U}$ such that $Y \times(X(S))^{n+1} \subset \bigcup \mathscr{U}^{\prime}$. Since $\mathscr{U}^{\prime}$ is countable, there is $\alpha<\omega_{1}$ such that

$$
\begin{aligned}
& \text { if } U=U_{1} \times \prod_{i=1}^{n+1} U_{2, i} \in \mathscr{U}^{\prime}, \text { where } U_{1} \subset Y \text { and } U_{2, i} \subset X\left(\omega_{1}\right) \\
& \text { for all } i \leq n+1 \text {, then } P_{\alpha}^{-1} P_{\alpha}\left(U_{2, i}\right)=U_{2, i} .
\end{aligned}
$$

Observe that from (9) it follows that

$$
\begin{aligned}
& \text { if } y \in Y \text { and } x=\left(x_{1}, \ldots, x_{n+1}\right) \in\left(\bigcup\left\{Z_{\gamma}: \gamma \in \omega_{1} \backslash \alpha\right\}\right)^{n+1}, \\
& \text { then }(y, x) \in \bigcup \mathscr{U}^{\prime} .
\end{aligned}
$$

Hence

$$
Y \times\left(X\left(\omega_{1}\right)_{X(S)}\right)^{n+1} \backslash \bigcup \mathscr{U}^{\prime} \subset \bigcup_{i=1}^{n+1} E_{i}
$$

where

$$
E_{i}=\left\{\left(y,\left(x_{1}, \ldots, x_{n+1}\right)\right) \in Y \times\left(X\left(\omega_{1}\right)_{X(S)}\right)^{n+1}: x_{i} \in \bigcup\left\{Z_{\gamma}: \gamma \leq \alpha\right\}\right\} .
$$

The space $E_{i}$ is a countable union of subsets that are Lindelöf by the inductive assumption. We conclude that $\mathscr{U}$ has a countable subcover. This completes the proof of Lemma 2.

To finish the proof of the fact that $Y \times\left(X_{n}\right)^{n}$ is Lindelöf for every hereditarily Lindelöf space $Y$ is it sufficient to note that $\left(X_{n}\right)^{n}$ is a finite sum of elements each of which is a continuous image of a space of the form $\left(X\left(\omega_{1}\right)_{X(S)}\right)^{n}$, where $S$ is a stationary subset of $\omega_{1}$ and to apply Lemma 2.

Remark 1. R. Pol pointed out to me that one can also apply similar technique to the topology defined by G. Kurepa on a Aronszajn tree (see [T]) in order to obtain another example of the kind described in this paper.

Remark 2. Using the pressing-down Lemma one can show that the product $\left(X_{n}\right)^{n+1}$ is not normal for all $n \in N$. 


\section{REFERENCES}

[A1] K. Alster, $A$ class of spaces whose Cartesian product with every hereditarily Lindelöf space is Lindelöf, Fund. Math. 114 (1981), 173-181.

[A2] _ A note on Michael's problem concerning the Lindelöf property in the Cartesian products, Trans. Amer. Math. Soc. 278 (1983), 369-375.

[A3] _ On Michael's problem concerning the Lindelöf property in the Cartesian products, Fund. Math. 121 (1984), 149-167.

[A4] - On a question of Archangelskij concerning Lindelöf spaces with countable pseudocharacter, Proc. Amer. Math. Soc. 95 (1985), 320-322.

[A5] _ On spaces whose product with every Lindelöf space is Lindelöf, Colloq. Math. 54 (1987), 171-178.

[A6] _ On the class of all spaces of weight not greater than $\omega_{1}$ whose Cartesian product with every Lindelöf space is Lindelöf, Fund. Math. 129 (1988), 133-140.

[E] R. Engelking, General topology, revised and completed edition, 1989.

[J] T. Jech, Lectures in set theory, Lecture Notes in Math., vol. 217, Springer-Verlag, Berlin, 1971.

[T] S. Todorcevic, On the Lindelöf property of Aronszajn trees, Proc. of the Sixth Topological Symposium 1986, Research and Exposition in Math., Berlin, 1988, pp. 577-588.

Institute of Mathematics of the Polish Academy of Sciences, Śniadeckich 8, 00-950 WARSAW, POLAND 